\title{
Reassessment of the mitochondrial 12S-rRNA gene for DNA barcoding of museum specimens of shelled marine gastropods from Japan
}

\author{
Nao Fukunaga ${ }^{1}$, Moe Shimizu ${ }^{1}$, Shinnosuke Teruya ${ }^{2}$, Nazifa Naziha Razali $^{1}$, Satoko \\ Nakashima ${ }^{1,4}$, Naoto Tomobuchi ${ }^{1}$, Takenori Sasaki ${ }^{3}$, and Davin H. E. Setiamarga ${ }^{1,3,{ }^{*}}$ \\ ${ }^{1}$ National Institute of Technology (KOSEN), Wakayama College, Gobo, Wakayama, Japan, 644-0023 \\ ${ }^{2}$ Okinawa Prefectural Deep-sea Water Research Center, Kumejima, Okinawa, Japan \\ ${ }^{3}$ The University Museum, The University of Tokyo, Bunkyo-ku, Tokyo, Japan, 133-0023 \\ ${ }^{4}$ Present address: Graduate School of Frontier Science, The University of Tokyo, Kashiwa-no-ha, \\ Chiba, Japan, 277-8561
}

\begin{abstract}
DNA barcoding is an effective and powerful tool for taxonomic identification and thus very useful for biodiversity monitoring. This study investigated the usefulness of the mitochondrial 12S-rRNA gene for the DNA barcoding of shelled marine gastropods. To do so, we determined partial 12S-rRNA sequences of 75 vouchered museum specimens from 69 species of shelled gastropods from Japan. The specimens have been identified morphologically, and natural history data catalog. Sequence analyses through BLAST searches, maximum likelihood phylogenetic analysis, and species delimitation analysis suggested that the 12S-rRNA gene is helpful for barcoding shelled marine gastropods. They thus could be helpful to complement barcoding studies using other markers such as COI. The analyses successfully confirmed all samples' identity at higher taxonomy (subfamily and above), but much less so at the species level. Our result thus also underlines the lingering problem of DNA barcoding: The lack of comprehensive reference databases of sequences. However, since we provided sequences of properly curated, vouchered museum specimens in this study, our result reported here has thus also helped to give taxonomically reliable reference sequences for biodiversity monitoring and identifications of shelled gastropods which include many important fisheries species.
\end{abstract}

\section{Introduction}

With its ca. 35,000 of recorded species, Gastropoda, a class of shelled mollusks (Conchifera), is one of the most prominent invertebrate groups, in which, during its long evolutionary history, has radiated and occupied a diverse array of ecological niches in marine, freshwater, and biofouling/invasive organisms [2]. Many shelled members of this group are also greatly influenced by the recent ocean acidification event caused by global warming [1] [3-4]. As such, it is essential to monitor the diversity of this taxa constantly.

\footnotetext{
* Corresponding author: davin@wakayama.kosen-ac.jp
} 
Recent development in DNA sequencing and the building up of DNA sequence databases have allowed for the usage of DNA sequences for the quick and effective identifications and classifications of samples collected from the field with relatively high accuracy, a method called "DNA barcoding" [5]. Further development of DNA sequencing technology (e.g., Next Generation Sequencing) has allowed for the development of a non-invasive method of biodiversity monitoring using DNA sequences, called the eDNA (environmental DNA = eDNA) method, which is essential barcoding of DNA fragments shed off into the environment by living organisms [6-8]. For DNA barcoding and e-DNA to work, the availability of a robust, reliable, and exhaustive reference database of DNA sequences collected from target taxa, is essential. However, at present, biases in biodiversity studies might have caused such a reliable database to be not available for some taxa, including gastropods (e.g. [9]). In addition, Machida et al. [10] and Page [11] reported that currently, some data are not adequately curated. As a result, some taxa might become unidentifiable and thus become "dark taxa."This becomes very problematic in monitoring studies, especially those conducted by people with inadequate taxonomical skills and resources, or if target taxa contain many possible undescribed or cryptic species, or when conducting eDNAbased monitoring for which morphological samples are simply unavailable.

On the technical side, the marker genes must have enough base substitutions to distinguish different species of the same genus [5], but not different enough to dramatically differentiate sympatric individuals of the same species. Several effective universal primers to amplify a region of the mitochondrial COI gene have been developed for metazoans, causing a tremendous amount of DNA barcoding studies to be conducted and thus empirically shown that the amplified COI segment has enough substitution rates to distinguish animals at the species level (e.g. [12-17]). However, in a previous study, we have suggested that doing DNA barcoding with only one genetic marker could be risky because of primarily technical problems (limited primer efficacies and the inability of a single quality to place samples properly at higher taxonomies) [16-17]. In that study [16-17], we also suggested that using multiple markers would help to alleviate the problems because it would allow for the collection of a more complete genetic data (primers of different genes might work on samples not amplifiable with those of one marker gene, and a combination of markers would allow for a more robust phylogeny), and thus allowing researchers to collect a more complete picture reflecting the actual biodiversity. Therefore, in that study, we evaluated and thus proposed the usefulness of the nuclear gene Histone- $\mathrm{H} 3(\mathrm{H} 3)$ as a marker for DNA barcoding of shelled gastropods, using previously developed primers [18].

In this study, to develop and assess the utility of another molecular marker for DNA barcoding-based studies of shelled gastropods, we investigated the usefulness of the mitochondrial gene 12S-rRNA using previously reported primers [19-20]. The 12S-rRNA gene has been used in previous molecular phylogenetics, phylogeography, and DNA barcoding of various metazoans, including gastropods. We sequenced the 12S-rRNA of different vouchered samples of shelled marine gastropods stored at the University Museum of The University of Tokyo, including some old museum samples (the oldest was sampled in 1999, and the latest was tested in 2015). Our result presented here, which was based on BLAST searches, phylogenetic analysis, and species delimitation analysis, has confirmed the usefulness of the 12S-rRNA as a genetic marker for DNA barcoding of shelled gastropods, which also include many fisheries species. The result also highlighted the lack of a robust and comprehensive reference database of this gene if it is to be used as a marker for gastropods. Meanwhile, because we used properly curated and vouchered museum specimens as samples, the natural history data of our representatives are reliable. Therefore, we have also contributed a set of reference sequence data from taxonomically reliable samples through this study, which is crucial for biodiversity monitoring using DNA barcoding and e-DNA. 


\section{Materials and methods}

\subsection{Sample collection}

A total of 75 individuals of 69 species of shelled gastropods were used in this study. All samples used in this study were vouchered specimens stored in the University Museum, The University of Tokyo. These samples were initially collected from various locations in Japan, and then fixed and stored in 95\% EtOH. Morphological identifications (based on [21]) of collected samples were conducted before or after fixation. Representatives were chosen at random, with one or two individuals per species. Most specimens are at least nine years old, with the most senior sample collected in 1999, and the latest in 2015. The list of samples is provided in Table 1.

\subsection{DNA sequencing and sequence data acquisition}

A piece of the muscle tissue from the mantle or the foot (about $0.25 \mathrm{mg}$ ) was cut out from each sample. Total genomic DNA was extracted using the standard CTABphenol-chloroform method. PCR was performed using a standard protocol but with an annealing temperature of $52^{\circ} \mathrm{C}$. Three combinations of previously published three primers (Table 2) [19-20] were used to amplify the 12S-rRNA fragment of samples. Sanger sequencing of amplicons (using both the forward and reverse primers) was outsourced (FASMAC Co. Ltd., Kanagawa, Japan). For comparison, we also sequenced a fragment of the COI gene of all samples, using previously published primers [22-24]. The list of primers used in this study is shown in Table 2. Obtained sequences were then checked for contamination by BLASTn searches [25]. Sequence fidelity was confirmed and edited manually on the software MESQUITE ver. 3.61 [26-27], by also simultaneously checking the chromatograms by eye (visualized on ApE ver.2.0.61 [28]). After sequence editing, all forward and reverse sequences were assembled manually by eye.

\subsection{Sequence identification (DNA barcoding) through BLASTn searches}

In order to confirm if the obtained sequences were homologous to previously published arrangements, and thus to get taxonomic information of the organisms from which the lines were obtained, we performed BLASTn searches on the assembled sequences. We consider a sample as correctly identified if the morphological identification matches the BLASTn search result. We also confirmed at which taxonomic level a particular sequence was identified (species, genus, family, order) to check the availability of reference sequences on GenBank and the fidelity of the GenBank sequences. BLASTn searches were conducted for both the 12S-rRNA and COI gene sequences. 
Table 1. List of analyzed OTU in this study, along with the BLASTn search results for DNA

\begin{tabular}{|c|c|c|c|c|c|c|c|}
\hline \multirow{2}{*}{ Sample No. } & \multirow{2}{*}{ Species } & \multicolumn{6}{|c|}{$12 \mathrm{~S}$} \\
\hline & & Genebank ID & BLASTn result & Homology Level & E-value & Identity (\%) & Gaps\% \\
\hline SS45 & Batillaria multiformis & NC 047187.1 & Batillaria attramentaria & Genus & 0 & 98 & 0 \\
\hline B308 & Batillaria zonalis & HQ833855.1 & Batillaria cumingi & Genus & 0 & 98 & 0 \\
\hline SS104 & Planaxis sulcatus & HQ833854.1 & Planaxis sulcatus & Species & 0 & 99 & 0 \\
\hline B309 & Semisulcospira libertina & NC 037771.1 & Semisulcospira coreana & Genus & $3.0 \mathrm{E}-160$ & 93 & 0 \\
\hline B303 & Cerithidea cingulata & AB535193.1 & Cerithidea djadjariensis & Genus & $8.0 \mathrm{E}-126$ & 88 & 3 \\
\hline SS57 & Nerita albicilla & LC215360.1 & Nerita albicilla & Species & 0 & 99 & 0 \\
\hline SS124 & Nerita helicinoides & MT161611.1 & Nerita chamaeleon & Genus & $6.0 \mathrm{E}-162$ & 94 & 0 \\
\hline SS59 & Nerita japonica & LC565707.1 & Nerita japonica & Species & 0 & 99 & 0 \\
\hline SS126 & homoiodoris japonica & MT161611.1 & Nerita chamaeleon & Genus & $1.0 \mathrm{E}-173$ & 96 & 0 \\
\hline SS120 & Nerita striata & KF728888.1 & Nerita fulgurans & Genus & $2.0 \mathrm{E}-162$ & 94 & 0 \\
\hline SS103 & Cassidula mustelina & KJ920319.1 & Cassidula nucleus & Species & $2.0 \mathrm{E}-171$ & 96 & 0 \\
\hline SS141 & Erronea errones & HQ833858.1 & Erronea errones & Species & 0 & 98 & 0 \\
\hline SS83 & Echinolittorina radiata & AJ623151.1 & Echinolittorina radiata & Species & 0 & 99 & 0 \\
\hline SS142 & Notocochlis gualteriana & MK507895.1 & Notocochlis sp. & Genus & 0 & 99 & 0 \\
\hline SS143 & Canarium mutabile & MW244820.1 & Tridentarius dentatus & Family & $1.0 \mathrm{E}-163$ & 94 & 0 \\
\hline SS110 & Conomurex luhuanus & KY853669.1 & Conomurex luhuanus & Species & 0 & 99 & 0 \\
\hline B349 & Mitrella burchardi & HQ833864.1 & Mitrella bicincta & Genus & 0 & 99 & 0 \\
\hline B366 & Fusinus ferrugineus & NC_ 045906.1 & Fusinus longicaudus & Genus & 0 & 98 & 0 \\
\hline SS115 & Pleuroploca trapezium & $\mathrm{MN} 322355.1$ & Turrilatirus turritus & Subfamily & $2.0 \mathrm{E}-137$ & 90 & 1 \\
\hline B355 & Nassarius conoidalis & NC_041310.1 & Nassarius conoidalis & Species & 0 & 99 & 0 \\
\hline B342 & Nassarius fraterculus & $\mathrm{NC}^{-} 037604.1$ & Nassarius fraterculus & Species & 0 & 99 & 0 \\
\hline SS102 & Nassarius albescens & KY4489008.1 & Nassarius fenistratus & Genus & 0 & 99 & 0 \\
\hline SS144 & Nassarius coronatus & KY488995.1 & Nassarius coronatus & Species & 0 & 99 & 0 \\
\hline SS44 & Cantharus mollis & HQ833883.1 & Cantharus cecillei & Genus & 0 & 97 & 0 \\
\hline B344 & Enzinopsis menkeana & FM999097.1 & Pisania striata & Family & $5.0 \mathrm{E}-158$ & 93 & 0 \\
\hline SS140 & Pterygia dactylus & KR087379.1 & Pterygia dactylus & Species & 0 & 100 & 0 \\
\hline SS123 & Coralliophila neritoidea & AJ293679.1 & Coralliophila neritoidea & Species & 0 & 98 & 0 \\
\hline SS106 & Drupella cornus & FR853980.1 & Drupella cornus & Species & 0 & 99 & 0 \\
\hline B356 & Oppomorus funiculatus & HE583824.1 & Morula funiculata & Species & 0 & 99 & 0 \\
\hline B318 & Nucella lima & KJ093800.1 & Nucella heyseana & Genus & 0 & 100 & 0 \\
\hline B336 & Ocenebra inornatus endermonis & NC 046052.1 & Ocinebrellus falcatus & Genus & 0 & 98 & 0 \\
\hline SS81 & Mancinella echinata & HE5 84089.1 & Mancinella echinata & Species & 0 & 99 & 0 \\
\hline SS70 & Mancinella siro & HE584090.1 & Mancinella grossa & Genus & 0 & 99 & 0 \\
\hline SS86 & Menathais tuberosa & KU747972.1 & Menathais tuberosa & Species & 0 & 99 & 0 \\
\hline B302 & Reishia bronni & NC 039165.1 & Thais luteostoma & Genus & 0 & 98 & 0 \\
\hline SS75 & Reishia bronni & HQ833878.1 & Thais luteostoma & Genus & 0 & 99 & 0 \\
\hline B293 & Thais clavigera & HE584119.1 & Reishia clavigera & Species & 0 & 99 & 0 \\
\hline SS34 & Thais clavigera & HE584119.1 & Reishia clavigera & Species & 0 & 99 & 0 \\
\hline
\end{tabular}


Table 1. (Continued).

\begin{tabular}{|c|c|c|c|c|c|c|c|}
\hline \multirow{2}{*}{ Sample No. } & \multirow{2}{*}{ Species } & \multicolumn{6}{|c|}{$12 \mathrm{~S}$} \\
\hline & & Genebank ID & BLASTn result & Homology Level & E-value & Identity (\%) & Gaps $\%$ \\
\hline SS116 & Vasum turbinellum & HQ833909.1 & Vasum turbinellus & Species & 0 & 99 & 0 \\
\hline B363 & Homoiodoris japonica & КР 635442.1 & Homoiodoris japonica & Species & 0 & 99 & 0 \\
\hline B323 & Acmaea pallida & MT370382.1 & Niveotectura pallida & Species & $5.0 \mathrm{E}-132$ & 93 & 0 \\
\hline B253 & Collisella dorsuosa & $\mathrm{AB} 106454.1$ & Lottia dorsuosa & Species & $1.0 \mathrm{E}-152$ & 99 & 0 \\
\hline B334 & Tectura emydia & MT370375.1 & Lottia instabilis & Genus & 0 & 99 & 0 \\
\hline B357 & Lepeta kuragiensis & $\mathrm{AB} 238235.1$ & Cryptobranchia kuragiensis & Species & $1.0 \mathrm{E}-149$ & 99 & 0 \\
\hline SS95 & Lottia luchuana & AB106453.1 & Lottia luchuana & Species & $2.0 \mathrm{E}-172$ & 99 & 0 \\
\hline B396 & Nipponacmea boninensis & KU316594.1 & Nipponacmea radula & Genus & 0 & 99 & 0 \\
\hline SS14 & Nipponacmea radula & KU316566.1 & Nipponacmea fuscoviridis & Genus & 0 & 100 & 0 \\
\hline S391 & Nipponacmea schrenkii & KU316582.1 & Nipponacmea schrenckii & Species & 0 & 96 & 0 \\
\hline SS17 & Patelloida pygmaea & $\mathrm{AB} 106436.1$ & Patelloida pygmaea & Species & $7.0 \mathrm{E}-161$ & 99 & 0 \\
\hline SS91 & Patelloida ryukyuensis & AB238280.1 & Patelloida ryukyuensis & Species & $2.0 \mathrm{E}-166$ & 100 & 0 \\
\hline B256 & Patelloida saccharina lanx & LC142820.1 & Nipponacmea boninensis & Family & $1.0 \mathrm{E}-163$ & 99 & 0 \\
\hline SS51 & Patelloida saccharina lanx & AB106439.1 & Patelloida saccharina lanx & Species & $8.0 \mathrm{E}-171$ & 100 & 0 \\
\hline SS2 & Cellana grata & GQ455887.1 & Cellana toreuma & Genus & 0 & 100 & 0 \\
\hline SS209 & Cellana grata & AB106427.1 & Cellana grata & Species & $3.0 \mathrm{E}-134$ & 99 & 0 \\
\hline SS18 & Cellana nigrolineata & LC600801.1 & Cellana nigrolineata & Species & $2.0 \mathrm{E}-141$ & 99 & 0 \\
\hline SS174 & Cellana radiata & AB106430.1 & Cellana radiata orientalis & Species & 2.0E-152 & 99 & 0 \\
\hline SS84 & Cellana testudinaria & AB106431.1 & Cellana testudinaria & Species & $1.0 \mathrm{E}-153$ & 100 & 0 \\
\hline SS3 & Cellana toreuma & GQ455887.1 & Cellana toreuma & Species & 0 & 99 & 0 \\
\hline SS4 & Cellana toreuma & GQ455860.1 & Cellana mazatlandica & Genus & 0 & 99 & 0 \\
\hline B247 & Scutellastra flexuosa & AF058183.1 & Scutellastra flexuosa & Species & $6.0 \mathrm{E}-117$ & 92 & 1 \\
\hline B257 & Siphonaria sirius & KF001136.1 & Siphonaria sp. & Genus & $1.0 \mathrm{E}-172$ & 100 & 0 \\
\hline B359 & Siphonaria sp. & KF001069.1 & Siphonaria sp. & Genus & $8.0 \mathrm{E}-81$ & 84 & 3 \\
\hline SS131 & Tectus pyramis & MF138911.1 & Tectus pyramis & Species & 0 & 97 & 0 \\
\hline B268 & Chlorostoma argyrostoma lischkei & HE800683.1 & Chlorostoma lischkei & Genus & $5.0 \mathrm{E}-167$ & 100 & 0 \\
\hline B285 & Chlorostoma lischkei & HE800683.1 & Chlorostoma lischkei & Species & 0 & 99 & 0 \\
\hline SS72 & Chlorostoma turbinatum & HE800683.1 & Chlorostoma lischkei & Genus & 0 & 99 & 0 \\
\hline SS73 & Tegula pfeifferi pfeifferi & NC_056356.1 & Omphalius rusticus & Genus & 0 & 98 & 0 \\
\hline SS67 & Omphalius nigerrimus & $\mathrm{NC}^{-} 031862.1$ & Omphalius nigerrimus & Species & 0 & 99 & 0 \\
\hline SS69 & Omphalius rusticus & $\mathrm{AF} 080631.1$ & Tegula rusticus & Species & 0 & 99 & 0 \\
\hline B254 & Cantharidus japonicus & AB505369.1 & Cantharidus jessoensis & Genus & 0 & 93 & 1 \\
\hline B345 & Cantharidus jessoensis & AB505369.1 & Cantharidus jessoensis & Species & 0 & 99 & 0 \\
\hline B332 & Lunella coreensis & MN604179.1 & Lunella correensis & Species & 0 & 99 & 0 \\
\hline SS54 & Lunella coreensis & MN604179.1 & Lunella correensis & Species & 0 & 99 & 0 \\
\hline SS101 & Lunella coronata & KX298890.1 & Lunella granulata & Genus & 0 & 99 & 0 \\
\hline B316 & Turbo stenogyrus & FR695555.1 & Turbo kenwilliamsi & Genus & 0 & 94 & 1 \\
\hline
\end{tabular}


Table 1. (Continued).

\begin{tabular}{|c|c|c|c|c|c|c|c|}
\hline \multirow{2}{*}{ Sample No. } & \multirow{2}{*}{ Species } & \multicolumn{6}{|c|}{ COI } \\
\hline & & Genebank ID & BLASTn result & Homology Level & E-value & Identity (\%) & Gaps $\%$ \\
\hline SS45 & Batillaria multiformis & AB845820.1 & Batillaria multiformis & Species & 0 & 99 & 0 \\
\hline B308 & Batillaria zonalis & MN389045.1 & Batillaria cumingii & Genus & 0 & 99 & 0 \\
\hline SS104 & Planaxis sulcatus & MT620956.1 & Planaxis sulcatus & Species & 0 & 99 & 0 \\
\hline B309 & Semisulcospira libertina & KM031760.1 & Semisulcospira libertina & Species & 0 & 98 & 0 \\
\hline B303 & Cerithidea cingulata & HE680370.1 & Cerithideopsilla cingulata & Species & 0 & 100 & 0 \\
\hline SS57 & Nerita albicilla & EU253356.1 & Nerita albicilla & Species & 0 & 97 & 0 \\
\hline SS124 & Nerita helicinoides & EU732252.1 & Nerita helicinoides & Species & 0 & 99 & 0 \\
\hline SS59 & Nerita japonica & EU732262.1 & Nerita japonica & Species & 0 & 98 & 0 \\
\hline SS126 & homoiodoris japonica & MW277894.1 & Nerita polita & Species & 0 & 99 & 0 \\
\hline SS 120 & Nerita striata & EU732335.1 & Nerita undata & Genus & 0 & 100 & 0 \\
\hline SS103 & Cassidula mustelina & MN389193.1 & Cassidula nucleus & Species & 0 & 99 & 0 \\
\hline SS141 & Erronea errones & MK507895.1 & Notocochlis sp. & - & 0 & 96 & 0 \\
\hline SS83 & Echinolittorina radiata & HM560004.1 & Echinolittorina radiata & Species & 0 & 99 & 0 \\
\hline SS142 & Notocochlis gualteriana & MK507895.1 & Notocochlis sp. & Genus & 0 & 95 & 0 \\
\hline SS143 & Canarium mutabile & DQ525218.1 & Strombus mutabilis & Species & 0 & 96 & 0 \\
\hline SS110 & Conomurex luhuanus & KY 853669.1 & Conomurex luhuanus & Species & 0 & 96 & 2 \\
\hline B349 & Mitrella burchardi & JN052989.1 & Mitrella bicincta & Genus & 0 & 100 & 0 \\
\hline B366 & Fusinus ferrugineus & HM180585.1 & Fusinus longicaudus & Genus & 0 & 100 & 0 \\
\hline SS115 & Pleuroploca trapezium & KT753962.1 & Pleuroploca trapezium & Species & 0 & 97 & 0 \\
\hline B355 & Nassarius conoidalis & KY783915.1 & Nassarius conoidalis & Species & 0 & 99 & 0 \\
\hline B342 & Nassarius fraterculus & KX069666.1 & Nassarius fraterculus & Species & 0 & 100 & 0 \\
\hline SS 102 & Nassarius albescens & KY499727.1 & Nassarius albescens & Species & 0 & 99 & 0 \\
\hline SS144 & Nassarius coronatus & KY451287.1 & Nassarius coronatus & Species & 0 & 97 & 0 \\
\hline SS44 & Cantharus mollis & JN053007.1 & Cantharus cecillei & Genus & $3.0 \mathrm{E}-89$ & 88 & 1 \\
\hline B344 & Enzinopsis menkeana & KX519514.1 & Engina lanceolata & Genus & 0 & 93 & 0 \\
\hline SS140 & Pterygia dactylus & KR087291.1 & Pterygia dactylus & Species & 0 & 96 & 0 \\
\hline SS123 & Coralliophila neritoidea & MG917504.1 & Coralliophila violacea & Species & 0 & 99 & 0 \\
\hline SS106 & Drupella cornus & FR853843.1 & Drupella cornus & Species & 0 & 99 & 0 \\
\hline B356 & Oppomorus funiculatus & HE584045.1 & Morula funiculata & Species & 0 & 99 & 0 \\
\hline B318 & Nucella lima & KJ093791.1 & Nucella heyseana & Genus & 0 & 99 & 0 \\
\hline B336 & Ocenebra inornatus endermonis & HM180657.1 & Lunella coreensis & - & 0 & 99 & 0 \\
\hline SS81 & Mancinella echinata & KC466605.1 & Mancinella echinata & Species & 0 & 97 & 2 \\
\hline SS70 & Mancinella siro & HE584344.1 & Mancinella siro & Species & 0 & 98 & 0 \\
\hline SS86 & Menathais tuberosa & KU747972.1 & Menathais tuberosa & Species & 0 & 99 & 0 \\
\hline B302 & Reishia bronni & HM180825.1 & Thais luteostoma & Genus & 0 & 99 & 0 \\
\hline SS75 & Reishia bronni & NC 039165.1 & Thais luteostoma & Genus & 0 & 99 & 0 \\
\hline B293 & Thais clavigera & HM1 180819.1 & Reishia clavigera & Species & 0 & 98 & 1 \\
\hline SS34 & Thais clavigera & MH400316.1 & Reishia clavigera & Species & 0 & 99 & 0 \\
\hline
\end{tabular}


Table 1. (Continued).

\begin{tabular}{|c|c|c|c|c|c|c|c|}
\hline \multirow{2}{*}{ Sample No. } & \multirow{2}{*}{ Species } & \multicolumn{6}{|c|}{$\mathrm{COI}$} \\
\hline & & Genebank ID & BLASTn result & Homology Level & E-value & Identity (\%) & Gaps $\%$ \\
\hline SS116 & Vasum turbinellum & HQ834084.1 & Vasum turbinellus & Species & 0 & 99 & 0 \\
\hline B363 & Homoiodoris japonica & KP635442.1 & Homoiodoris japonica & Species & 0 & 99 & 0 \\
\hline B323 & Acmaea pallida & LC416617.1 & Niveotectura pallida & Species & 0 & 97 & 0 \\
\hline B253 & Collisella dorsuosa & KM221108.1 & Lottia dorsuosa & Species & 0 & 91 & 0 \\
\hline B334 & Tectura emydia & MT814212.1 & Lottia instabilis & Genus & 0 & 99 & 0 \\
\hline B357 & Lepeta kuragiensis & AB543974.1 & Cryptobranchia kuragiensis & Species & 0 & 99 & 0 \\
\hline SS95 & Lottia luchuana & AB238471.1 & Lottia luchuana & Species & 0 & 99 & 0 \\
\hline B396 & Nipponacmea boninensis & LC383956.1 & Japeuthria ferrea & - & 0 & 99 & 0 \\
\hline SS14 & Nipponacmea radula & KC 844158.1 & Nipponacmea fuscoviridis & Genus & 0 & 99 & 0 \\
\hline S391 & Nipponacmea schrenkii & FR693994.1 & Lunella coreensis & - & 0 & 100 & 0 \\
\hline SS17 & Patelloida pygmaea & AB238519.1 & Patelloida pygmaea & Species & 0 & 98 & 0 \\
\hline SS91 & Patelloida ryukyuensis & AB238520.1 & Patelloida ryukyuensis & Species & 0 & 99 & 0 \\
\hline B256 & Patelloida saccharina lanx & HM180776.1 & Patelloida saccharina lanx & Species & 0 & 99 & 0 \\
\hline SS51 & Patelloida saccharina lanx & HM180776.1 & Patelloida saccharina lanx & Species & 0 & 99 & 0 \\
\hline SS2 & Cellana grata & HM180722.1 & Notoacmea schrenckii & - & 0 & 100 & 0 \\
\hline SS209 & Cellana grata & KM221072.1 & Cellana grata & Species & 0 & 98 & 0 \\
\hline SS18 & Cellana nigrolineata & LC600801.1 & Cellana nigrolineata & Species & 0 & 100 & 0 \\
\hline SS174 & Cellana radiata & AB238554.1 & Cellana radiata orientalis & Species & 0 & 99 & 0 \\
\hline SS84 & Cellana testudinaria & AB238563.1 & Cellana testudinaria & Species & 0 & 99 & 0 \\
\hline SS3 & Cellana toreuma & HM180724.1 & Notoacmea schrenckii & - & 0 & 100 & 0 \\
\hline SS4 & Cellana toreuma & KM221072.1 & Cellana grata & Genus & 0 & 99 & 0 \\
\hline B247 & Scutellastra flexuosa & KT149318.1 & Scutellastra flexuosa & Species & 0 & 87 & 0 \\
\hline B257 & Siphonaria sirius & KF000832.1 & Siphonaria sp. & Genus & 0 & 99 & 0 \\
\hline B359 & Siphonaria sp. & MF652008.1 & Siphonaria fuegiensis & Genus & $1.0 \mathrm{E}-175$ & 84 & 0 \\
\hline SS131 & Tectus pyramis & MN388983.1 & Tectus pyramis & Species & $6.0 \mathrm{E}-177$ & 95 & 0 \\
\hline B268 & Chlorostoma argyrostoma lischkei & EU530144.1 & Chlorostoma lischkei & Genus & 0 & 99 & 0 \\
\hline B285 & Chlorostoma lischkei & EU530145.1 & Chlorostoma lischkei & Species & 0 & 99 & 0 \\
\hline SS72 & Chlorostoma turbinatum & LC413975.1 & Chlorostoma turbinatum & Species & 0 & 99 & 0 \\
\hline SS73 & Tegula pfeifferi pfeifferi & HM180731.1 & Omphalius pfeifferi carpenteri & Species & 0 & 99 & 0 \\
\hline SS67 & Omphalius nigerrimus & HE800629.1 & Tegula aff. Argyrostoma & Genus & 0 & 98 & 0 \\
\hline SS69 & Omphalius rusticus & NC_056356.1 & Omphalius rusticus & Species & 0 & 99 & 0 \\
\hline B254 & Cantharidus japonicus & EŪ530120.1 & Cantharidus callichroa & Genus & 0 & 90 & 0 \\
\hline B345 & Cantharidus jessoensis & AB505280.1 & Cantharidus jessoensis & Species & 0 & 100 & 0 \\
\hline B332 & Lunella coreensis & MN604179.1 & Lunella correensis & Species & 0 & 99 & 0 \\
\hline SS54 & Lunella coreensis & MN604179.1 & Lunella correensis & Species & 0 & 99 & 0 \\
\hline SS101 & Lunella coronata & KX298890.1 & Lunella granulata & Genus & 0 & 99 & 0 \\
\hline B316 & Turbo stenogyrus & AM403915.1 & Turbo stenogyrus & Species & 0 & 99 & 0 \\
\hline
\end{tabular}


Table 2. List the 12S-rRNA and COI gene primers used in this study.

\begin{tabular}{llll}
\hline Gene & \multicolumn{1}{c}{ Primer } & \multicolumn{1}{c}{ Sequence(5'-3') } & Reference \\
\hline \multirow{2}{*}{$12 \mathrm{~S}$} & 12Sma $(\mathrm{F})$ & CTGGGATTAGATACCCTGTTAT & {$[19]$} \\
& 12S97L (F) & AACYCAAAGRACTTGGCGGT & {$[20]$} \\
& 12Smb (R) & CAGAGAGTGACGGGCGATTTGT & {$[19]$} \\
COI & LCO1490 (F) & GGTCAACAAATCATAAAGATATTGG & {$[22]$} \\
& LCOmod (F) & TCTACTAATCATAAGGAYATYGGNAC & {$[24]$} \\
& HCO2198 (R) & TAAACTTCAGGGTGACCAAAAAATCA & {$[22]$} \\
& H7005 (R) & CCGGATCCACNACRTARTANGTRTCRTG & {$[23]$} \\
& HCOmod (R) & ACTTCTGGGTGTCCRAARAAYCARAA & {$[24]$} \\
\hline
\end{tabular}

\subsection{Sequence editing, dataset preparation, and phylogenetic analysis}

Confirmed, edited, and assembled sequences were aligned using MAFFT v7 [29]., the dataset containing aligned sequences was edited in Gblocks 0.91b (Online ver.) to exclude ambiguously aligned regions [30]. Furthermore, visualized in Mesquite ver. 3.61 [26-27]. Maximum likelihood (ML) phylogenetic inference was conducted in RAxML-GUI v.1.5 [31] under the GTR-GAMMA substitution model, partitioned per-gene, with 1000X bootstrap samplings carried out to assess the robustness of the obtained topology. Model selection before phylogenetic analysis was carried out in MEGA X [32]. Two Bivalves, Pinctada fucata (AB250258.1) and Crassostrea gigas (EF484878.1), were used as outgroups. Phylogenetic analysis was conducted only on the 12S-rRNA gene sequences.

\subsection{Species delimitation analysis}

Species delimitation analyses were conducted on the sequence datasets of COI and 12SrRNA using the Automatic Barcode Gap Discovery (ABGD) software [33] to see if the 12SrRNA fragment was used in our barcoding could differentiate species]. The analyses used the aligned sequence data of the 12S-rRNA (length $=280 \mathrm{bp}$ ) and COI (length $=632 \mathrm{bp}$ ). Prior intraspecific divergence range (Pmin to Pmax) was set to $0.001-0.1$, and the $\mathrm{X}$ value for the minimum relative gap width was set to 0.99 under the K2P model with TS/TV $=2.0$.

\section{Results}

\subsection{Sequence data acquisition}

For the 12S-rRNA gene marker, we successfully amplified and thus obtained ca. $450 \mathrm{bp}$ for 12Sma / 12Smb primer pairs (60 samples) and ca. $430 \mathrm{bp}$ of 12S97L / 12Smb primer pairs (15 samples), making us successfully obtain the 12S-rRNA sequences of all individuals used in this study. After sequence editing and alignments, the 12S-rRNA sequence lengths used for phylogenetic analysis were $280 \mathrm{bp}$. We also successfully amplified the COI gene marker for all samples in this study (ca. 650 bp for LCO1490 / HCO2198 primer pairs = 54 samples; ca. 1100 bp for LCO1490 / H7005 primer pairs = 6 samples; ca. 650 bp for LCOmod_Kano2008 / HCOmod_Kano2008 primer pairs = 15 samples). The sequence length of the $\mathrm{COI}$ marker after sequence editing and alignments was $632 \mathrm{bp}$. 


\subsection{BLASTn searches-based DNA barcoding}

BLASTn searches of the gene 12S-rRNA matched 39 species of 41 individuals (Table 1), with the sequence identities of $92 \%-100 \%$ and e-values of 0.00 to 6.00 e- 117 . Meanwhile, 30 samples of 29 species were confirmed only at the genus level (22 genera). The rest of the samples (four individuals) matched Genbank sequences at higher taxonomy (i.e., subfamily, family, and order levels). Meanwhile, for the COI gene, 47 species (50 individuals; 67\%) were confirmed at the species level, with 25 individuals matched at the taxonomic levels of genera and above. Detailed results of the BLASTn searches are presented in Table 1.

\subsection{Obtained phylogeny and the taxonomic placement of the samples}

The phylogenetic tree obtained from the maximum likelihood phylogenetic inference performed on the 12s-rRNA sequences is shown in Figure 1. Most samples were placed along with their morphologically identified species and genera levels with relatively reasonable support values (e.g., Cellana, $\mathrm{BS}=72 \%$; Chlorostoma, $\mathrm{BS}=70 \%$; Nerita, $\mathrm{BS}=53 \%$ ). However, Nassarius and Patelloida were not monophyletic, although both genera were placed in their proper higher taxonomies (Nasarius: Buccinoidea; Patelloida: Lottiidae). Placements at the higher taxonomy levels (subfamily and above) for other genera and species also placed most of them in their valid taxa, despite the lack of strong bootstrap support on many clades, and improper taxonomic placements of some samples (Figure 1), for example, members of Muricoidea, Nucella lima, and Ocenebra inornatus endermonis, were placed in Buccinoidea, while Notocochlis gualteriana (Naticoidea) was placed in Muricoidea; the Cypraeoid Erronea errones was placed in Buccinoidea; some members of Littorinimorpha, Erronea errones, Notocochlis gualteriana, Canarium mutabile, Conomurex luhuanus, were instead put inside Neogastropoda, and Cassidula mustelina (Ellobiida) was included in Neogastropoda.

\subsection{Species delimitations of target samples}

Species delimitations conducted in ABGD on the 12S-rRNA gene dataset resulted in the identification of 61 species, while the same analysis on the dataset of the COI gene identified 65 species (Table 3). Meanwhile, our samples (75 OTUs) were morphologically identified as 69 species. As shown in Table 3, the result of ABGD on both genes generally agrees with the outcome of morphological identification. However, some closely related congeneric species were not properly delimited and identified as the same species in one of the markers or both. For example, Batillaria multiformis and Batillaria zonalis were identified as one species by the 12s-rRNA, while Cellana grata and Cellana toreuma were identified as one species by both gene markers. 


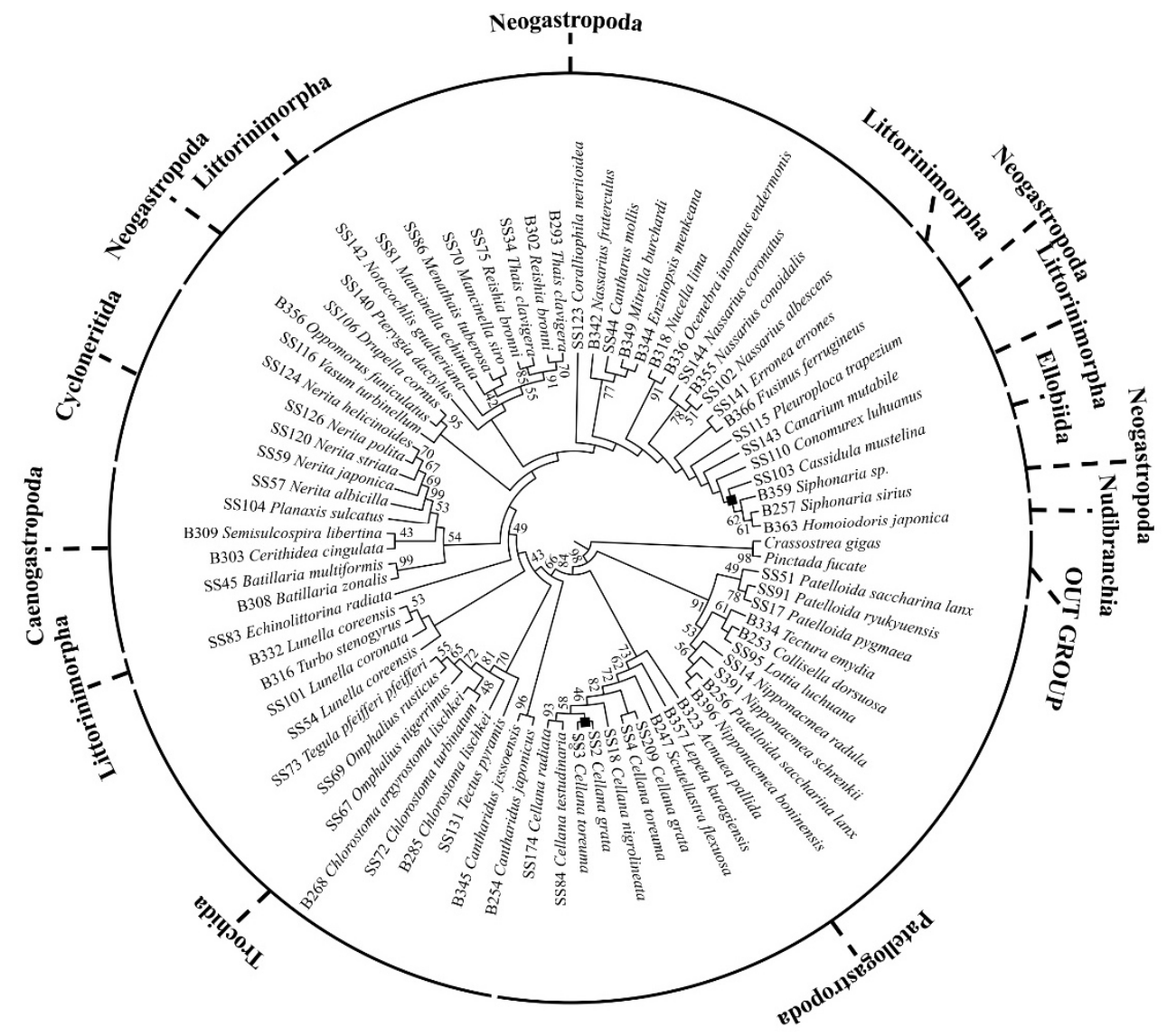

Fig. 1. Maximum likelihood phylogenetic tree of our samples based on 12S-rRNA sequences obtained in this study. Numbers on a branch denote bootstrap value of an adjacent node. Bootstrap values lower than $41 \%$ are not written, while fully supported nodes are denoted by

\section{Discussion}

\subsection{Sequence data acquisition success rate on preserved museum samples}

In this study, we tested the 12S-rRNA primers on various museum samples across the whole Gastropods. The usefulness of the COI primers has been shown in multiple previous studies [13-14], including those of ours [16-17]. In general, our results here indicate that the 12SrRNA primers tested in this study are helpful and can amplify the 12S-rRNA sequence fragments for DNA barcoding to complement results obtained using other markers such as COI. Meanwhile, we successfully got DNA sequences of both gene markers for all samples using standard PCR protocols, even though some of the samples were old museum samples, which were stored at conditions not ideal for molecular works (e.g., room temperature storage). Therefore, our result also suggests that these primers could probably be used for museums studies, such as museum samples barcoding and studies to obtain molecular data out of old museum samples [34]. 


\subsection{The non-exhaustiveness of Genbank for the identification of gastropods}

We performed BLASTn searches of the COI and 12S-rRNA sequences obtained from the samples to see if the sequences of our taxa are present on Genbank, besides checking if our morphological identification was consistent with the sequence data on Genbank. The result of our BLASTn searches suggested that most samples only $55 \%$ of our samples were correctly identified using the 12S-rRNA and only $67 \%$ even when using COI, which is the most commonly used DNA barcoding marker [35]. This result thus underlines the problem of taxonomic bias in biodiversity observation, causing the incompleteness and/or nonexhaustiveness of the data base [9]. This could be problematic for studies depending on identification based on DNA sequences only, such as eDNA and metagenomics [6].

\subsection{The phylogeny is relatively well-resolved for a single marker}

We also conducted a phylogenetic analysis of the 12S-rRNA sequences to see if the gene could adequately place the samples in their proper taxa. We found that while most samples were properly grouped with their conspecifics or congeners, some samples were not (Figure 1). At present, we are unable to pinpoint the cause of these misplacements, which might include sequence errors, homoplasies and long-branch attractions, and the possible lack of identifying substitutions of the 12S-rRNA fragment used in our phylogenetic analysis. Meanwhile, classification at higher taxonomy, in general, is congruent with the recently proposed gastropod systematics [36-37]. This result is generally also in agreement with the result of our preliminary study [38]. However, detailed interrelationships did not agree entirely, and the statistical supports were low in most nodes of higher taxonomy. It is expected since the interrelationships among higher taxa (above genus) cannot usually be resolved using only single-gene data [16-17,38-39].

\subsection{The 12S-rRNA marker was able to delimit most species in this study}

We also conducted species delimitation analyses on the COI and 12S-rRNA to confirm if both characteristics could correctly identify/delimit the species of the samples, as identified morphologically. There was 69 morphospecies (out of 75 individuals) in our samples, which were identified by professional taxonomists/curators, which were also co-authors, of this study. Interestingly, however, both markers were unable to completely delimit all morphospecies $(\mathrm{COI}=65$ species, but $12 \mathrm{~S}=71$ species), apparently having difficulties differentiating closely related congeneric species (Table 3 ).

The differences in species delimitation could probably be attributed to differences in the substitution rates of each taxon, which might be related to their different biology. This was also suggested by the species delimitation results of both genes before the removal of ambiguously aligned regions by GBlocks. These results indicated that the removal of ambiguously aligned regions might affect the detection of sequence diversity due to the removal of possible informative areas [40]. However, all in all, our result also indicated that both markers could delimit the samples at least at the genus level.

\subsection{General conclusion and future directions}

Our present results of species delimitation analysis, phylogenetic analysis, and BLASTn searches suggest that the short fragment of the 12S-rRNA gene used in this study is useful and effective enough to delimit various gastropod species, and thus useful for DNA barcoding and metabarcoding (eDNA studies) of shelled marine gastropods. The marker could thus be used to complement other DNA barcoding markers such as COI and 18S-rRNA. DNA 
barcoding using multiple markers would allow researchers to capture a complete snapshot of biodiversity and avoid the numerous possible pitfalls caused by using only a single marker [16-17, 41-44].

Moreover, because our study presented appropriately used curated museum samples, our sequence data would become an essential addition to the reference database for future studies. Therefore, in the future, we will register our sequence data to an adequately curated database such as Genbank or DDBJ. We will also continue our study by testing more prospective markers on more properly curated museum samples of shelled marine gastropods from Japan to provide a comprehensive reference sequence database for further studies involving DNA barcoding, metabarcoding, and e-DNA.

\section{Acknowledgements}

We would like to thank previous and present members of the Setiamarga Lab at National Institute of Technology, Wakayama College, and the Sasaki Lab at The University Museum, The University of Tokyo, for constant support during this study. This study was financially supported by Nakatsuji Foresight Foundation FY2018 Research Grants for Basic Sciences [to DHES], FY2019 JSPS KAKENHI Grant-in-Aid for Scientific Research (C) Grant No. 19K12424 [to DHES and TS], and JSPS KAKENHI for Challenging Research (Exploratory) Grant No. 19K21646 [to TS and DHES]. 
Table 3. Results of analysis of 12S-rRNA and COI using ABGD.

\begin{tabular}{|c|c|c|c|c|}
\hline \# & Sample No. & Morphological & $12 \mathrm{~S}$ & $\mathrm{COI}$ \\
\hline 1 & SS45 & Batillaria multiformis & \multirow{2}{*}{$12 S \_$species 1} & COI species 1 \\
\hline 2 & B308 & Batillaria zonalis & & COI species 2 \\
\hline 3 & SS104 & Planaxis sulcatus & $12 S$ species 2 & COI species 3 \\
\hline 4 & B309 & Semisulcospira libertina & $12 S$ species 3 & COI species 4 \\
\hline 5 & B303 & Cerithidea cingulata & $12 S$ species 4 & COI species 5 \\
\hline 6 & SS57 & Nerita albicilla & $12 S$ species 5 & COI species 6 \\
\hline 7 & SS124 & Nerita helicinoides & \multirow{2}{*}{$12 S \_$species 6} & COI species 7 \\
\hline 8 & SS120 & Nerita striata & & COI species 8 \\
\hline 9 & SS59 & Nerita japonica & $12 S$ species 7 & COI species 9 \\
\hline 10 & SS126 & Homoiodoris japonica & $12 S$ species 8 & COI_species 10 \\
\hline 11 & SS103 & Cassidula mustelina & $12 S$ species 9 & COI species 11 \\
\hline 12 & SS141 & Erronea errones & $12 S$ species 10 & \multirow{2}{*}{ COI_species 12} \\
\hline 13 & SS142 & Notocochlis gualteriana & $12 S$ species 11 & \\
\hline 14 & SS83 & Echinolittorina radiata & $12 S$ species 12 & COI species 13 \\
\hline 15 & SS143 & Canarium mutabile & $12 S$ species 13 & COI species 14 \\
\hline 16 & SS110 & Conomurex luhuanus & $12 \mathrm{~S}$ species 14 & COI species 15 \\
\hline 17 & B349 & Mitrella burchardi & $12 S$ species 15 & COI species 16 \\
\hline 18 & B366 & Fusinus ferrugineus & $12 S$ species 16 & COI species 17 \\
\hline 19 & SS115 & Pleuroploca trapezium & $12 S$ species 17 & COI species 18 \\
\hline 20 & B342 & Nassarius fraterculus & $12 S$ species 18 & COI species 19 \\
\hline 21 & B355 & Nassarius conoidalis & \multirow{3}{*}{$12 S \_s p e c i e s 19$} & COI species 20 \\
\hline 22 & SS102 & Nassarius albescens & & COI_species 21 \\
\hline 23 & SS144 & Nassarius coronatus & & COI species 22 \\
\hline 24 & SS44 & Cantharus mollis & $12 S$ species 20 & COI_species 23 \\
\hline 25 & B344 & Enzinopsis menkeana & $12 S$ species 21 & COI species 24 \\
\hline 26 & SS140 & Pterygia dactylus & $12 S$ species 22 & COI_species 25 \\
\hline 27 & SS123 & Coralliophila neritoidea & $12 S$ species 23 & COI species 26 \\
\hline 28 & SS106 & Drupella cornus & $12 S$ species 24 & COI species 27 \\
\hline 29 & B356 & Oppomorus funiculatus & $12 S$ species 25 & COI species 28 \\
\hline 30 & B318 & Nucella lima & \multirow{2}{*}{$12 S_{\text {_species }} 26$} & COI species 29 \\
\hline 31 & $\mathrm{~B} 336$ & Ocenebra inornatus endermonis & & COI species 30 \\
\hline 32 & SS81 & Mancinella echinata & $12 S$ species 27 & COI_species 31 \\
\hline 33 & SS70 & Mancinella siro & $12 S$ species 28 & COI_species 32 \\
\hline 34 & SS86 & Menathais tuberosa & $12 S$ species 29 & COI_species 33 \\
\hline $\begin{array}{l}35 \\
36\end{array}$ & $\begin{array}{l}\text { B302 } \\
\text { SS75 }\end{array}$ & Reishia bronni & \multirow{2}{*}{$12 S \_s p e c i e s 30$} & COI_species 34 \\
\hline $\begin{array}{l}37 \\
38\end{array}$ & $\begin{array}{l}\text { B293 } \\
\text { SS34 }\end{array}$ & Thais clavigera & & COI_species 35 \\
\hline 39 & SS116 & Vasum turbinellum & $12 S$ species 31 & COI species 36 \\
\hline 40 & B363 & Homoiodoris japonica & $12 S$ species 32 & COI species 37 \\
\hline
\end{tabular}


Table 3. (Continued).

\begin{tabular}{|c|c|c|c|c|}
\hline \# & Sample No. & Morphological & $12 \mathrm{~S}$ & $\mathrm{COI}$ \\
\hline \multicolumn{5}{|c|}{ Acmaea pallida } \\
\hline \multirow{2}{*}{$\begin{array}{l}42 \\
43\end{array}$} & \multirow{2}{*}{$\begin{array}{l}\text { B253 } \\
\text { B334 }\end{array}$} & Collisella dorsuosa & $12 S$ species 34 & COI species 39 \\
\hline & & Tectura emydia & $12 S$ species 35 & COI species 40 \\
\hline \multirow{2}{*}{$\begin{array}{l}44 \\
45\end{array}$} & B357 & Lepeta kuragiensis & $12 S$ species 36 & COI species 41 \\
\hline & SS95 & Lottia luchuana & $12 S$ species 37 & COI species 42 \\
\hline \multirow{2}{*}{$\begin{array}{l}46 \\
47\end{array}$} & B396 & Nipponacmea boninensis & $12 S$ species 38 & COI species 43 \\
\hline & \multirow{4}{*}{$\begin{array}{l}\text { SS14 } \\
\text { SS17 } \\
\text { SS91 } \\
\text { B256 }\end{array}$} & Nipponacmea radula & $12 S$ species 39 & COI species 44 \\
\hline \multirow{3}{*}{$\begin{array}{l}48 \\
49 \\
50 \\
\end{array}$} & & Patelloida pygmaea & $12 S$ species 40 & COI species 45 \\
\hline & & Patelloida ryukyuensis & $12 S_{\text {sppecies }} 41$ & COI_species 46 \\
\hline & & Patelloida saccharina lanx & $\begin{array}{l}12 S_{\text {_species }} 42 \\
12 S_{\text {species }} 43\end{array}$ & COI_species 47 \\
\hline \multirow{3}{*}{$\begin{array}{l}51 \\
52 \\
53 \\
54\end{array}$} & \multirow{2}{*}{$\begin{array}{l}\text { SS51 } \\
\text { SS2 } \\
\text { SS3 }\end{array}$} & Cellana grata & $12 S_{\text {species } 44}$ & \multirow{2}{*}{ COI_species 48} \\
\hline & & Cellana toreuma & I2S_species 44 & \\
\hline & \multirow{6}{*}{\begin{tabular}{|l} 
SS209 \\
SS4 \\
SS18 \\
SS174 \\
SS84 \\
B247 \\
B257
\end{tabular}} & Cellana grata & \multirow[b]{2}{*}{$12 S \_$species 45} & COI_species 49 \\
\hline \multirow{5}{*}{$\begin{array}{l}55 \\
56 \\
57 \\
58 \\
59 \\
60\end{array}$} & & $\begin{array}{l}\text { Cellana toreuma } \\
\text { Cellana nigrolineata }\end{array}$ & & \begin{tabular}{|l|} 
COI species 50 \\
\end{tabular} \\
\hline & & $\begin{array}{l}\text { Cellana nigrolineata } \\
\text { Cellana radiata }\end{array}$ & $12 S$ species 46 & COI species 51 \\
\hline & & Cellana testudinaria & $12 S$ species 47 & COI species 52 \\
\hline & & Scutellastra flexuosa & $12 S$ species 48 & COI species 53 \\
\hline & & Siphonaria sirius & $12 S$ species 49 & COI species 54 \\
\hline \multirow{2}{*}{$\begin{array}{l}61 \\
62\end{array}$} & \multirow{2}{*}{$\begin{array}{l}\text { B359 } \\
\text { SS131 }\end{array}$} & Siphonaria sp. & $12 S$ species 50 & COI species 55 \\
\hline & & Tectus pyramis & $12 S$ species 51 & COI species 56 \\
\hline \multirow{2}{*}{\multicolumn{2}{|c|}{\begin{tabular}{l|l}
63 & B268 \\
64 & SS72
\end{tabular}}} & Chlorostoma argyrostoma lischkei & \multirow{2}{*}{$12 S \_$species 52} & \multirow{3}{*}{ COI_species 57} \\
\hline & & Chlorostoma turbinatum & & \\
\hline 65 & B285 & Chlorostoma lischkei & $12 S$ species 53 & \\
\hline \multirow{5}{*}{$\begin{array}{l}66 \\
67 \\
68 \\
69 \\
70\end{array}$} & \multirow{5}{*}{$\begin{array}{l}\text { SS73 } \\
\text { SS69 } \\
\text { SS67 } \\
\text { B254 } \\
\text { B345 }\end{array}$} & Tegula pfeifferi pfeifferi & $12 S$ snecies 54 & COI species 58 \\
\hline & & Omphalius rusticus & I2S_species 54 & COI species 59 \\
\hline & & Omphalius nigerrimus & $12 S$ species 55 & COI species 60 \\
\hline & & Cantharidus japonicus & & COI species 61 \\
\hline & & Cantharidus jessoensis & $12 S_{\text {_species }} 56$ & COI species 62 \\
\hline 71 & S391 & Nipponacmea schrenkii & $12 S$ species 57 & \\
\hline 72 & B332 & Lunella coreensis & $12 S \_$species 58 & COI_species 63 \\
\hline $\begin{array}{l}13 \\
74\end{array}$ & $\begin{array}{l}\text { SS54 } \\
\text { SS101 }\end{array}$ & Lunella coronata & $\frac{12 S \text { species } 59}{12 S \text { species } 60}$ & COI species 64 \\
\hline 75 & B316 & Turbo stenogyrus & $12 S$ species 61 & COI species 65 \\
\hline & & 69 & 61 & 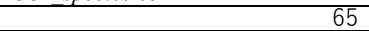 \\
\hline
\end{tabular}




\section{References}

1. W. Appeltan, S.T. Ahyong, G. Anderson, M.V. Angel, T. Artois, N. Bailly, et al, Curr. Biol. 22, 23 (2012)

2. W.F. Ponder, D.R. Lindberg, J.M. Ponder, Biology and evolution of the Mollusca: volume two (CRC Press, Florida, 2019)

3. J.C. Orr, V.J. Fabry, O. Aumont, L. Bopp, S.C. Doney, R.A. Feely, et al., Nature 437, 681-686 (2005)

4. R. Rodolfo-Metalpa, F. Houlbaèque, É. Tambutté, F. Boisson, C. Gabbini, F.P. Patti, et al, Nature Clim. Change 1, 308-312 (2011)

5. P.D.N. Hebert, A. Cywinska, S.L. Ball, J.R. deWaard, Royal Soc. B. 270, 1512 (2003)

6. P.F. Thomsen, E. Willerslev, Biol. Conserv. 183, 4-18 (2015)

7. K. Deiner, H.M. Bik, E. Mächler, M. Seymour, A. Lacoursière-Roussel, F. Altermatt, et al, Mol. Ecol. 26, 21 (2017)

8. M.E. Cristescu, P.D.N. Hebert, Annu. Rev. Ecol. Evol. Syst. 49, 209-230 (2018)

9. J. Troudet, P. Grandcolas, A. Blin, R. Vignes-Lebbe, F. Legendre, Sci. Rep. 7, 9132 (2017)

10. R.J. Machida, Y. Hashiguchi, M. Nishida, S. Nishida, BMC Genom. 10, 438 (2009)

11. R.D.M. Page, Philos. Trans. R. Soc. Lond. B. Biol. Sci. 371, 1702 (2016)

12. O. Folmer, M. Black, W. Hoah, R. Lutz, R. Vrijenhoek, Mar. Biotechnol. 3, 294-299 (1994)

13. Y. Kano, Zool. Scr. 37, 1-20 (2008)

14. LMS. Borges, C. Hollatz, J. Lobo, A.M. Cunha, A.P. Vilela, G. Calado, et al, Sci. Rep. 6, 20226 (2016)

15. S. Zou, Q. Li, L. Kong, H. Yu, X. Zheng, PLoS ONE 6, 10, (2011)

16. N. Nakaji, S. Iwamoto, S. Teruya, M. Kusube, A. Kosaka, T. Sasaki, et al, SEE 2018 , 28-44 (2018)

17. D.H.E. Setiamarga, N. Nakaji, S. Iwamoto, S. Teruya, T. Sasaki, Int. J. GEOMATE, 17, 62 (2019)

18. D.J. Colgan, A. McLauchlan, G.D.F. Wilison, S.P. Livingston, G.D. Edgecombe, J. Macaranas, et al., Aust. J. Zool. 46, 5 (1998)

19. V. Koufopanou, D.G. Reid, S.A. Ridgway, R.H. Thomas, Mol. Biol. Evol. 70, 1 (1999)

20. T. Nakano, T. Ozawa, J. Molluscan Stud. 70, 1 (2004)

21. T. Okutani, Marine mollusks in Japan (in Japanese) (The Association of Japanese University Press, Tokyo, 2017)

22. O. Folmer, M. Black, W. Hoah, R. Lutz, R Vrijenhoek, Mar. Biotechnol. 3, 5 (1994)

23. M.S. Hafner, P. D. Sudman, F.X. Villablanca, T.A. Spradling, J.W. Demastes, S.A. Nadler, Science 265, 5175 (1994)

24. Y. Kano, Zool. Scr. 37, 1 (2008)

25. S.F. Altschul, W. Gish, W. Miller, E.W. Myers, D.J. Lipman, J. Mol. Biol. 215, 3 (1990)

26. W. Maddison, D. Maddison, Evolution 11, 5 (2009)

27. W.P. Maddison, D.R. Maddison, Mesquite: a modular system for evolutionary analysis, Version $3.61 \mathrm{http}: / /$ www.mesquiteproject.org (2018) 
28. M.W. Davis, ApE-A plasmid editor, https://jorgensen.biology.utah.edu/wayned/ape/

29. K. Katoh, J. Rozewicki, K.D. Yamada, Brief. Bioinf. 20, 4 (2019)

30. J. Castresana, Mol. Biol. Evol. 17, 4 (2002)

31. D. Silvestro, I. Michalak, Org. Divers. Evol. 12, 4 (2012)

32. S. Kumar, G. Stecher, M. Li, C. Knyaz, K. Tamura, Mol. Biol. Evol. 35, 6 (2018)

33. N. Puillandre, A. Lambert, S. Brouillet, G.J.M.E. Achaz, Mol. Ecol. 21, 8 (2012)

34. D.H.E. Setiamarga, R. Shiba, Y. Kamito, M. Yamamoto, N.N.B. Razali, M. Arai, et al, Zoosymposia 15, 129-140 (2019)

35. P.D.N. Hebert, M.Y. Stoeckle, T.S. Zemlak, C.M. Francis, C. Godfray, PLOS Biol. 2, 10 (2004)

36. T. Sasaki, Malacology (In Japanese) (The University of Tokyo Press, Tokyo, 2010)

37. T.J. Cunha, G. Giribet, Proc. Royal Soc. B, 286, 1898 (2019)

38. S. Nakashima, N. Nakaji, D.V. Tu, T. Sasaki, D.H.E. Setiamarga, Proc. 28th OES, 1-6 (2020)

39. A. Som, Brief. Bioinf. 16, 3 (2015)

40. F. Lutzoni, P. Wagner, V. Reeb, S. Zoller, Syst. Biol. 49, 4 (2000)

41. H. Song, J.E. Buhay, M.F. Whiting, K.A. Crandall, Proc. Natl. Acad. Sci., 105 (2008)

42. M. Miya, R.O. Gotoh, T. Sado, Fish. Sci. 86, 1-32 (2020)

43. E. Valsecchi, J. Bylemans, S.J. Goodman, R. Lombardi, I. Carr, L. Castellano, et al., Environ. DNA 2, 4 (2020)

44. F. De Mattia, R. Gentili, I. Bruni, A. Galimberti, S. Sgorbati, M. Casiraghi, et al, Bot. J. Linn. Soc. 169, 3 (2012) 